\title{
DIECISIETE CASOS DE MICETOMAS ACTINO Y EUMICOTICOS EN ROSARIO, ARGENTINA (1959 - 1998)
}

\author{
Seventeen cases of actino and eumycotic mycetoma in \\ Rosario, Argentina (1959-1998)
}

\author{
Carlos R. Gómez \& Marisa S. Biasoli \\ Centro de Referenciá de Micología, Facul tad de Ciencias \\ Bioquímicas y Farmacéulicas. Universidad Nacional de Rosario. \\ Suipacha 531-(2000). Rosario, Argentina \\ E-mail cgomez@ fbioyf.unr.edu.ar
}

Palabras clave: Micetomas, actinomicóticos, elımicóticos Key words: Mycetoma,actinomy'colics, eumycotics

\section{RESUMEN}

Se comminican 17 casos de miceromas estudiados en el periodo comprendido entre Enero de 1958 y: Noviembre de 1998,10 éunicóticos (59\%) y 7 actinomicólicos (H1\%). Madurella grisea fue el agente más aislack (29.5\%) en los primeros y Nocardia asteroides (17.6\%) en los segumdos: Los agentes restanles fueron Psendallescheria boidii, Madurella mycetomatis, Fusarium solani, Nocardia brasiliensis y Actinomadura madurae. A pesar que los micelomas som afeciciones benignas, las dificullades terapémicas los transforman en cuadros climicos más serios:

\section{INTRODUCCION}

El micetoma es un pseudolumor inflamatorio crónico, destmclor, progresivo, localizado y no contagioso que afecta la piel, fascia. tẹido celular subcutáneo, músculo y ocasionalmente huesos y tejidos adyacentes. Puede ser causado tanto por un agente fúngico o un aclinomiceto aeróbico, los cuales son introducidos clentro de la dermis por un traumatismo (1,2). La localización más frecuente es el pie y por eso la enfermedad a menudo es conocicla como "Pie de Madura", sin embargo. exislen olras localizaciones como las manos o sitios sujelos a traumatismos (3). Cuando su origen es fúngico. se los denomina Eumycelomas o nucetomas maduromicóticos y Actinomiceloma, cuando es produciclo por un aclinomiceto aeróbico cle los géneros

\section{SUMMARY}

The present 17 cases of mycetoma studied from January 1958 10 November 1998. Ten cases (59\%) were consed by true fungi, while seven (11\%) were caused by Actinomycates. Among enmycetoma Madurella grisca Has isolated from five cases (29.5\%), Nocardia asteroides, the most common actinomycete isolated, was responsible for three cases. The rest of the agents were: Pseudullescheria boidii, Malurella mycetomatis, Fusarium solani, Nocardia brasiliensis y Actinomadura madurae. Myceloma is a benigh affection, however therapeulic difficulties make it a serious condition, having a severe functional proginosis.

Nocardia, Actinomadura o Streptomyces. $(4,5,6)$ Eslc síndrome se caracteriza por tumefacción, deformación y aumento de tamaño de la zona afectada, dando aspeclo pscudolumoral, con producción de nódulos, abscesos, zonas de fibrosis de consisiencia leñosa y trayeclos fistulosos. que tienen la capacidad de penetrar hasta el tejido profundo y drenar hacia el exterior un material punulento o serosanguinolento: con granos de distintos colores, forma: y consistencias, que son agregados vegetativos del agente Causall $(7,8,9,10)$. El tiempo de evolución de un miceloma es muy variabley depende del microor-ganismo y del paciente mismo. Los primeros signos clásicos pueden apurecer varios meses y aún años después del traumatismo infeclante. Luego el micetoma crece lentamente durante uno a varios años a veces liasla $2 i$ a a $25(3)$. 
Las características histopatológicas halladas y la identificación microbiológica del agente, conducen al diagnóslico. Es muy importante establecer el agente causal, ya que de esto depende la conducia terapéulica a seguir.

En Argentina el mayor número de casos se produce en el norte del país, en zonas de clima subtropical.

El objetivo del presente trabajo es actualizar los datos sobre micetomas observados en nuestra región y compararlos con otros datos del país y del mundo.

\section{MATERIALES Y METODOS}

En el laboratorio del Centro de Referencia de Micología (CE.RE.MIC.), en el período comprendido entre enero de 1958 y noviembre de 1998, se procesaron 51.641 muestras clínicas de pacientes para estudios micológicos. Del total de estos, se consideraron para eldiagnóstico de micetoma, aquellos pacientes que presentaron la triada clínica de nódulos subcutáneos, presencia de fistulas y descarga de gránulos (7).

Los materiales analizados en este grupo de pacientes se basaron en: biopsias de lejidos subculáneos y profundos incluyendo los trayectos fistulosos y secreciones purulentas o sero-sanguinolentas que drenan de las fistulas.

Hasta el año 1980, no hay datos acerca de la metodología utilizada para la determinación del agente etiológico y por esta razón, se consignaron solamente dalos estadísticos basados en los registros de resultados. Luego de ese año, la determinación del agente etiológico se basó en :

1) Aspectos macroscópicos del grano parasitario al fresco, describiendo su tamaño, color, forma y consistencia.

2) Aspectos microscópicos del grano en montajes con $\mathrm{KOH}$ al $20 \%$ y cortes histológicos teñidos con hematoxilina-eosina. Gram-Nicolle y Kinyoun, describiendo la morfología del gránulo, el tamaño de los fílamentos, la presencia de sustancia cementante. la presencia de pigmentos en la pared fúngica y la afinidad por los colorantes.

Sobre la base de la observación microscópica se clasificaron como eumicóticos aquellos que presentaron filamentos fúngicos labicados de 2 a 4 pm de diámetro o como actinomicól icos, si presentaban filamentos muy finos no tabicados de $1 \mu \mathrm{m}$ o menos.

3) Estudios micológi cos o bacteriológicos a partir de los cultivos obtenidos.

Lavado de los gránulos actinomicóticos varias veces con solución fisiológica (SF) estéril y siembra en medio de Lowenslein-Jensen, caldo tioglicolato, agar blando glucosaclo ( $0.3 \%$ agar y $2 \%$ dextrosa), Czapek con anzuelo de parafina e incubación a $37^{\circ} \mathrm{C}$.

Lavado de los grannulos eumicóticos con SF estéril con estreptomicina y cloranfenicol y posterior cultivo en agar Sabouraud glucosa (Sb), Sb con cloranfenicol (Sbcl) y agar cerebro corazón con cloranfenicol $(\mathrm{CCcl})$ a 28 y 37 ${ }^{\circ} \mathrm{C}$. En ambos casos la incubación es de 3 a 6 semanas.

Con los desarrollos obtenidos a partir de cultivos, en los casos de los micetomas eumicóticos, se realizaron estudios macro y micromorfológicos, y' en los casos de los actinomicóticos, estudios micromorfológicos de las caracteristicas culturales y las siguientes pruebas bioquímicas: reducción de nitrato, producción de ureasa, crecimiento en gelatina al $0,4 \%$, descomposición cle caseína, tirosina, xantina, hipoxantina, hidrólisis de la gelatina y almidón, producción de ácidos a partir de distinlas sustancias hidrocarbonadas, tolerancia a distintas temperaturas y acido resistencia (Rippon,1982)(11). Esıas técnicas fueron utilizadas desde 1980. Previo a este año, la identificación se basó en: morfologia del micelio, acido resistencia, desarrollo en parafina como única fuente de carbono, desarrollo en agua gelatinada al 0.4\% hidrólisis de ovoalbúmina, gelatina y caseina; degradación de xantina $y$ tirosina.

\section{RESULTADOS}

En el período estudiaclo se detectaron solo 17 casos de micetomas (un promedio de 0,425 casos por año o 0,33 casos $/ 1000$ consultas). Se produjeron 10 casos de micetomas eumicólicos, lo que corresponde al $59 \%$ y 7 actinomicóticos $(41 \%)$.

Los eumicetomas fueron causados por Mudurella grisea en 5 casos (29,5\%). Pseudoallescheria boydii en 3 casos (17.6\%), Madurella mycetomatis y Fusarium

Tabla 1:Agentes etiológicos de 17 casos de micetoma en el CE.RE.MIC

\begin{tabular}{|lcr|}
\hline AGENTE & $\mathbb{N}^{0}$ de casos & $\%$ \\
\hline EUMICETOMA: & 10 & $\mathbf{5 9 , 0}$ \\
Madurella grisea & 5 & 29,5 \\
Pseudoallescheria boydii & 3 & $\mathbf{1 7 , 6}$ \\
Madurella mycetonatis & 1 & 5,9 \\
Fusarium solani & 1 & 5,9 \\
\hline & & \\
ACTINOMICETOMA: & 7 & 41,0 \\
Nocardia asteroides & 3 & 17,6 \\
N.brasiliensis & 2 & 11,7 \\
Actinomadura madurae & 1 & 5,9 \\
Actinomicete & 1 & 5,9 \\
\hline TOTAL & 17 & $\mathbf{1 0 0 , 0}$ \\
\hline
\end{tabular}


solani, en un caso cada uno (Tabla l). En la tabla 2 se detallan las características más importantes de 6 de estos 10 pacientes; los datos indicados corresponden a los cásos más recientes, todos despues de 1980, ya que de los anteriores no fue posible contar con los registros de todos los datos que se indican en la tabla. Los micetomas actinomicóticos fueron causados por Nocardia asteroides en 3 casos (17,6\%), N. brasiliensis en 2 (11,7\%), Actinomadura madurac en uno (5,9\%) y un caso de un actinomycete que no pudo ser aislado (5.9\%) (Tabla 1).

\section{DISCUSION}

Este grupo de pacientes no representa la incidencia real de los micetomas en la Argentiná, pero el interés de este estudio fue el de contribuir a un mejor conociniento de su epidemiología en el país. El período estudiado comprende un lapso de 40 años en donde el número de casos de micetomas detectados (diecisiele), es realnente muy bajo con respecto a la incidencia en otras regiones de América y del mundo.

En nuestro país, Negroni et al. (12), presentaron 54 casos de micelomas en un período de 10 años, con el $51,9 \%$ de eumicetomas y $48,1 \%$ de actinomicetomas.

En América; los países con mayor número de casos cle micelomas son México. Brasil, Colombia y' Venezuela (13).

En Brasil. Castro et al.(14)detecla ron. enl un período de 12 años, 41 casos de micetomas (con un promedio de $0.5 / 1000$ consultas), de las cuales el $32 \%$ fíreron eumicóticos y el $68 \%$ actinomicóticos. En ese mismo país. Lacaz et al. (citado en Castro el al., ), en un período de 34 años, diagnosticó 154 casos cle micetomas y al igual que los otros autores, el mayor porcentaje correspondió a micetomas aclinomicólicos.

En México, que es el país que presenta mayor número de casos en Aunéricá, López Mártínez et al.(15), en un período de 30 años, clescribieron 2105 casos, con una incidencia de 70 cásos por año. El 97,8\% correspondió a micetomas actinomicóticos. con el mayor porcentaje debido a Nocardia brasiliensis ( $86.6 \%$ ) y solo el $2.2 \%$ de eumicetomas debidos a Madurella grisea y M. nycetomotis.

Otros países que cuentan con un alto número de casos son Sudán. Senegal, Somalia. India, Indonesia y Pakistán $(7,16)$. Todas estas regiones del mundo, son endémicas para esta enfermedald y se caracterizan por tener: un clima tropical o subtropical con un período corto de lluvias de 4 a 6 meses, una temperatura de 30 a $37^{\circ} \mathrm{C}$, una lummedad relativa de 60 a $80 \%$ con un período seco de 6-8 meses ( $T^{\circ} 45-60^{\circ} \mathrm{C}$ durante el día y $15-18^{\circ}$ durante la noche) y una liumedad relativa de 12 a $30 \%(3,17)$.

Nuestra región, no presenta estas características, ya que el clima es más bien templado, pero hay que destacar que la mayoría de los pacientes a los cuales se les diagnosticó miceloma, soll provenientes de otras zonas del país, generalmente de la región del norte argentino, que sí responde a las características climáticas antes menciomadas (12).

Con respecto a la localización y el sexo, los eumicetomas, en nuestro estudio, al igual que en la literatura $(16,17)$, fueron observados exclusivamente en el pie y en el $100 \%$ de los casos en pacientes del sexo masculino. Estos hechos pueden estar relacionados con la actividad laboral ya que, en su mayoría, se desempeñaban en tareas rurales. En cuanto a la edad de nuestros pacientes, esta osciló entre 24 y 59 años. Aunque la enfermedad se encuentra en todos los grupos de edades. la atención clínicá es solicitada sólo después de varios años de desarrollo (tiempo de evolución entre 6 meses y 35 años); así el rango en que se presenta la enfermedad con más frecuencia es entre los 30 y 50 años $(11,16,17)$ (Tabla 2).

Los agentes etiológicos del micetoma varían de acuerdo con el área del mundo estudiada. Madurella mycetomatis es, según Mc Ginnis (17), el lıongo productor de eumicetomas más común en todo el mundo. Según el mismo autor, otros agentes son en orden de frecuencia: Pseudoallescheria boydii, Leptosphaeria senegalensis, Madurella grisea, Acremonium recifei, etc. Algunos de estos agentes son geográficamente importantes productores de micetomas, como $\boldsymbol{M}$. grisea en Sudamérica. $P$. boydii en zonas de clima templado en los Estados Unidos, Europa (18) y en nuestra región. Esle último hongo, según Castro et al. (14), es un agente común en los países no endémicos, donde los eumicetomas son observados esporádicamente, a diferencia de los países endémicos de Africa, donde M. mycetomatis es el agente etiológico más frecuente. En muestro medio es Madurella grisea, mientras que Fusarium solani es muy poco frecuente (19).

Entre los aclinomicelomas, Nocardia asteroides y $N$. brasiliensis, fueron los agentes más frecuentemente aisládos entre los pacientes de nuestro estudio. En Brasil (14). México (15, 20), Venezuela (13) y Estados Unidos (18), $N$. brasiliensis es el agente más frecuente, mientras que en los países de Africa y' Asia donde la enfermedad es endéluica, predominan las especies de Streptomyces y Actinomadura (7.16).

El micetoma en todas sus fases es sensible al tratamiento médico sólo o combinado con una cirugía reductora de masa, eliminando tejido infectado, pero debe evitarse la amputación o la desarticulación. El éxito del tratamiento depende no sólo de la diferenciación entre actinomicetoma y un eumicetoma sino también en lá identificación definitiva del agente causal $(17,21)$.

La enfermedad actinomicótica, responde al trata- 


\section{Tabla 2. Principales características de 6 pacientes masculinos con eumicetoma podal}

\begin{tabular}{|c|c|c|c|c|c|c|}
\hline Casos & $\mathbb{E d a d}$ & Residencia & Ocupación & $\begin{array}{l}\text { Tiempo } \\
\text { evolución }\end{array}$ & $\begin{array}{c}\text { Caract. del } \\
\text { gránulo }\end{array}$ & Agente etiológico \\
\hline 1 & 59 & $\begin{array}{l}\text { Stgo del Estero } \\
\text { / Chaco (NE) }\end{array}$ & Trabajo Rural & --- & $\begin{array}{c}\text { Blando, blanco } \\
\text { lobulado, } 0,5-1 \\
\mathrm{~mm}\end{array}$ & $\begin{array}{c}\text { Pseudoallescheria } \\
\text { boydii }\end{array}$ \\
\hline 2 & 28 & $\begin{array}{l}\text { Formosa } \\
\text { (NE) }\end{array}$ & $\begin{array}{l}\text { Tareas } \\
\text { agricolas }\end{array}$ & 2 años & $\begin{array}{c}\text { Negro, } \\
\text { semiblando, } \\
0,5-1 \mathrm{~mm}\end{array}$ & Madurella grisea \\
\hline 3 & 37 & $\begin{array}{l}\text { Entre Rios } \\
\text { (E) }\end{array}$ & $\ldots$ & $\begin{array}{c}10 \text { años } \\
\text { (comprom iso } \\
\text { oseo) }\end{array}$ & $\begin{array}{c}\text { Negro, } \\
\text { lobulado hasta } \\
1,5 \mathrm{~mm}\end{array}$ & $\begin{array}{l}\text { Madurella } \\
\text { mycetomatis }\end{array}$ \\
\hline 4 & 24 & $\begin{array}{c}\text { Chaco } \\
\text { (NE) }\end{array}$ & $\begin{array}{l}\text { Transportista } \\
\text { de madera }\end{array}$ & 3 meses & $\begin{array}{c}\text { Blanco, blando, } \\
1 \mathrm{~mm}\end{array}$ & Fuscrium soluni \\
\hline 5 & 35 & $\cdots N E$ & --- & --- & $\begin{array}{l}\text { Negro, duro, } \\
0,5 \mathrm{~mm}\end{array}$ & Madurella grisea \\
\hline 6. & 27 & $\begin{array}{l}\because \text { Chaco } \\
\quad(\dot{N E})\end{array}$ & Trabajo rural & 10 años. & $\begin{array}{c}\text { Negro, duro, } \\
0.5-1 \mathrm{~mm}\end{array}$ & Madurella grisea \\
\hline
\end{tabular}

$(\mathrm{NE})=$ Nordeste,$(\mathrm{E})=$ Este

miento con más facilídad que los micelonas eumicóticos (11:22), en esto radica la importancia del examen direclo del gránulo: Los nucclonas aclinomicóticos aún en eslado avanzado, responden bieri al tratamiento médico. Las drogas usadas incluyen: dapsona, streptomicina, sul lametoxazol-trimetoprima, tetraciclina. clindamicina y eritromicina: El último recurso cs la amputación, la cual está contraindicadá. pues favorcce las mctástasis o la disemunación hematógena $(14,18,22,23,24)$.

Los micetomas eumicóticos siempre son más resisientes a la quimioterapia dáclo que los niveles de la drogá en la zona de là lesión, suelen ser bastante más bajos que los requeridos para inhibir al agente participante (11). Entre las drogas usadas se nombran: anfotericina B, itraconazol, ketonconazol y griseofulvina. El éxilo en el tratamiento está relacionado con la extensión de la afección; en los casos muy avanzados se aplica la cirugía de amputación quilando también tejido sano $(14,22,23)$. Los tratamientos deben ser aplicados por tiempos prolongados y controlar los pacientes por más de 4 años (25). EJ miceloma es una afección benigna, no obstante lñas dificultades terapéuticas lo transforman en una afección seria con compromiso funcional grave (26).

\section{REFERENCIAS}

1-Ndiaye, B.; Develoux, M. \& Dieng, N.T. (1995). Les mycelomes a la clinique dermatologique de Dakar (Sencgal). Aspects Epidemiologiques, a propos de 111 eas. Ned. Afi. Noire 42:207-212

2- Kwon-Chung, K..J. \& Bennett, J.F. (1992). Medical Mycology: Philadelphia. P.A. La \& Febiger pp.560-593

3- Nariat, F. (1975). Los Micelomas. In: CIC, Publicacion especial de las VI Jomadas Argentinas de Micologia. Buenos Aires pp.49-53

4- Gómez, C. (1995). Miceloma podal a Madmella grisen. Acta de resimenes VII Congreso sugenlino de Micologia, XVII Jormadas Argentinas de Micologia Rosario, Argentina pp.101-102

5- Mujica, M.T.; Biasoli M.S. \& Bracalenti B.J.C. (1984). Aistamiento y estudio de f'seadallescheria boylii de un micetoma podnl. Rev. Arg. Mlicol. 7:21-23
6- Biasoli, M. S.; Alvarez, D. P. \& Bracalenti, B. J. C. (I986). Sudy of a Mallarella grisea strain isolated fiom a fool myedoma. Mycopathologia 94:117-121

7- Fahal, A. H. \& Hassan, M. A. (1992). Mycetoma. Br. I. Surg. $79: 1138-1141$

8- Negroni, R. (1993). Significado y alcances de la palabra micelomals. Mélodos de estudio de este sindrome. Rev. Arg. Nicologia $14: 3-10$

9- Ramos, L; Luque, A. \& Alvarez, D. (1986). Aislamiento o identiticación de Madurella my'cetomatis de un miceloma podal. Bul. Nlicológico $3: 5-8$

10- Mc Elroy, J. A.; De Almeida-Prestes, J. \& Su, WV. P. (1992) Mycetoma: infection witl tumefaction. dratining sinuses, and grains. Culis 40:107-10 
11- Rippon, .J.W. (1982). Medical Mycology: The Pathugentistungi and Actinomreles. $2^{\text {mid }}$ E.d. Philadelphial: W. B. Saunders.pp. 91-132

12- Negroni, R.; HI lou, S.: Arechavala, A.; Robles, A.; Biancini, A. (1998). Micetomas en el Hospital Muñiz. Actal de resumenes vill Congreso Argentino de Nicologia. Tueumin pp.125

13- Rios Fabra, A.; Restrepo, A. \& Istúriy, R.E. (1994). Fungal infection in Latin American coumtries. Inlece. Dis. Clin. Nortls. Am. 8:129-34

14-Cinstro.l. C. M.; Bcha, W.; Salebian, A.; Cucé, L.C. (1993). Nefectoma: a retrospective study of 41 calses secol in bas Paulo. Braisil. from I978 (1) 1989. Mreoses 36:89-95

15- Lipe\% Martinez, R.; Mendez Tovar, I. J.: Lavalle, P.; Wolsh, O.: Situl. A.: Macotela, R.; Rui\%, li. (1992). Lipidemiologia del miceloma en Mexico: estudio de 2105 casos. (ialc. Med Miex 128:477-81

16- Venugopal, P. I. \& Vonugopal, T. I. (199)) . Acrinomndura madurae catusing mycetomals in Madras. Inelian I. Pathol. Microbiol $34: 119-25$

17- Mc Cimnis, M.R. (1996). Mycetoma. Dermatol. Clin. 14:97104

18- Ajello, L. (1978). MNectomats in the ('nited Stales. A critical

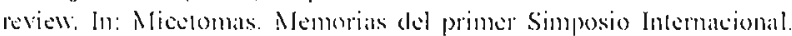
Carals. Ventezulit. pp.252-268

19- Lutuc, L.; Mujica, M. 'I. \& D'Anna, L. (1991). Mic心mal podal por Fusarium solumi. Bol. Micol. 6:55-57
20- Davila del Real, N.; Arenas, R.; Salazar, J.J.; Suaire\% de la Torre, R.: Campos, P.; Garcia, S.; R'adilla, M.; Bonifaz, A. (1996). Micetomas en el esrado de Guanajuato. Dermatol. Reve. Mex. 40:408-11

21- Mandell, G.L.; Douglas, R.G. \& Bennett, J.E. (1990). Principles and Practice of Infections Diseases. $3^{\text {rit }}$ Edition Chuchill Livingsiune Inc. New lork. pp. 2092-2096

22- Altma, D.T.; Lubahn, J.D.; Kulm, P..J.: Erie, P.A. (1994). A calse Report and Review of Aycetoma of the Hand: A Diagnostic and therapeutic Challenge. The Journal of liand surgery 19:A: 9981002

23- Restrepo, A. (1994). Treatment of tropical mycoses. I. Am. Acald. Dermalol. 3: S91-Siol

24-Ndiaye, B.; Develoux, M.; I,anglade, A.; Kanc, A. K. (1994). Les mycétomes Aetinomycosiques. A propos de 27 observations diakaroises, tratement medical par le cotrimoxazole. -tmales de dermalologia et de venérólogie. 2:161-165

25- Cindice, P' (1998). Treatment of eumyectomal. Journal of the Americin Academy of" Dermalology 39:137

26- Sounissi, R.; II amida, F.B \& Kamoun, N. (1990). Fóme parliculieremem extensive de Mycelome à atinomadura Madurate La Tunisic Médicale 68:633-636 